\title{
Nickel-based phosphide superconductor with infinite-layer structure, $\mathrm{BaNi}_{2} \mathbf{P}_{2}$
}

\author{
Takashi Mine ${ }^{1}$, Hiroshi Yanagi ${ }^{1}$, Toshio Kamiya ${ }^{1,2}$, Yoichi Kamihara ${ }^{2}$, Masahiro Hirano ${ }^{2,3}$, and \\ Hideo Hosono $1,2,3, *$ \\ ${ }^{1}$ Materials and Structures Laboratory, Tokyo Institute of Technology, 4259 Nagatsuta, Midori-ku, \\ Yokohama 226-8503, Japan \\ ${ }^{2}$ ERATO-SORST, JST, in Frontier Research Center, Tokyo Institute of Technology, 4259 Nagatsuta, \\ Midori-ku, Yokohama 226-8503, Japan \\ ${ }^{3}$ Frontier Research Center, Tokyo Institute of Technology, 4259 Nagatsuta, Midori-ku, Yokohama \\ 226-8503, Japan
}

\begin{abstract}
Analogous to cuprate high- $T_{\mathrm{c}}$ superconductors, a NiP-based compound system has several crystals in which the Ni-P layers have different stacking structures. Herein, the properties of $\mathrm{BaNi}_{2} \mathrm{P}_{2}$ are reported. $\mathrm{BaNi}_{2} \mathrm{P}_{2}$ has an infinite-layer structure, and shows a superconducting transition at $\sim 3 \mathrm{~K}$. Moreover, it exhibits metallic conduction and Pauli paramagnetism in the temperature range of $4-300 \mathrm{~K}$. Below $3 \mathrm{~K}$, the resistivity sharply drops to zero, and the magnetic susceptibility becomes negative, while the volume fraction of the superconducting phase estimated from the diamagnetic susceptibility reaches $\sim 100$ vol. $\%$ at $1.9 \mathrm{~K}$. These observations substantiate that $\mathrm{BaNi}_{2} \mathrm{P}_{2}$ is a bulk superconductor.
\end{abstract}

Keywords: A. Superconductor; D. Electronic transport

PACS: 74.25.Fy, 74.25.Ha, 74.70.Dd

Footnotes:

(*) Tel: $\quad+81-45-924-5359$

FAX: +81-45-924-5339

E- mail: hosono@msl.titech.ac.jp 


\section{Introduction}

$\mathrm{Cu}$ - and other transition metal-based compounds are attractive for exploring high-transition-temperature (high- $T_{\mathrm{c}}$ ) superconductors because it is considered that the high transition temperatures benefit from the strong electron correlation between $3 d$ electrons in the transition metal atoms. Many cuprate high- $T_{\mathrm{c}}$ superconductors have been discovered, including one reported in 1993, which has $T_{\mathrm{c}}$ of $133 \mathrm{~K}$ [1]. On the other hand, typical $T_{\mathrm{c}}$ 's of other transition metal based compounds (including oxides and pnictides) are lower than those of cuprate high- $T_{\mathrm{c}}$ superconductors. However, the discovery of new classes of superconductors, such as $\mathrm{Sr}_{2} \mathrm{RuO}_{4}$ [2], $\mathrm{Na}_{\mathrm{x}} \mathrm{CoO}_{2} \cdot \mathrm{yH}_{2} \mathrm{O}$ [3], electron-doped $\mathrm{HfNCl}$ [4], $\mathrm{Li}_{\mathrm{x}} \mathrm{NbO}_{2}$ [5], has provided complementary insight to better understand the mechanism of superconductivity as well as clues for exploring higher- $T_{\mathrm{c}}$ materials.

Recently, we have studied a series of quaternary compounds, which contain transition metal ions, $\mathrm{La} T_{M} \mathrm{OPn}\left(T_{M}=\right.$ transition-metal cations such as $\mathrm{Mn}, \mathrm{Fe}, \mathrm{Ni}$, and $\mathrm{Co} ; \mathrm{Pn}=\mathrm{P}$ and $\left.\mathrm{As}\right)$, with the expectation that this series will be a new correlated electron system. In our studies, we have found new superconductors, $\mathrm{LaFeOPn}[6,7]$ and $\mathrm{LaNiOPn}[8,9]$, and an itinerant ferromagnet, $\mathrm{LaCoOPn}$ [10]. As shown in Fig. 1(a), $\mathrm{La}_{M} \mathrm{O} P n$ has a layered crystal structure where the positively charged La-O layers and negatively charged $T_{M}-P n$ layers, which are composed of an edge-sharing network of $T_{M} P n_{4}$ tetrahedra, are alternately stacked along the $c$ axis. It is thought that the $T_{M}-P n$ layers sandwiched between the wider-gap La-O layers form magnetic and carrier conduction layers.

Ternary compounds containing transition metal ions, $A T_{M 2} \mathrm{P}_{2}(A=\mathrm{Ca}, \mathrm{Sr}, \mathrm{Ba}$, and lanthanide cations), have the $\mathrm{ThCr}_{2} \mathrm{Si}_{2}$ structure and belong to the $14 / \mathrm{nmm}$ space group. In addition, these ternary compounds show unusual physical properties such as intermediate valence states $\left(\mathrm{EuNi}_{2} \mathrm{P}_{2}[11]\right)$ and various magnetic properties, ranging from Pauli paramagnetism $\left(\mathrm{CaNi}_{2} \mathrm{P}_{2}\right.$ [12]) to ferromagnetism $\left(\mathrm{LaCo}_{2} \mathrm{P}_{2}[11]\right)$, and to antiferromagnetism $\left(\mathrm{CaCo}_{2} \mathrm{P}_{2}\right.$ [13]). Figure 1 (b) shows the crystal structure of $A T_{M 2} \mathrm{P}_{2}$, which has a layered structure similar to $\mathrm{La} T_{M} \mathrm{OP}$. The structure of the $T_{M}$-P layer is essentially the same as that in $\mathrm{La}_{M} \mathrm{OP}$ where the $T_{M}$-P layers are composed of an edge-sharing network of $T_{M} \mathrm{P}_{4}$ tetrahedra. However, the wider-gap La-O layers in $\mathrm{La} T_{M} \mathrm{OP}$ are replaced with the $A$ cation layers in $A T_{M 2} \mathrm{P}_{2}$. Thus, this crystal structure lacks a wider-gap insulating layer. Therefore, this structure may be regarded as an infinite-layer structure, which is analogous to cuprate high- $T_{\mathrm{c}}$ superconductors. Because an infinite-layer cuprate, $\left(\mathrm{Sr}_{1-\mathrm{x}} \mathrm{Ca}_{\mathrm{x}}\right)_{1-\mathrm{y}} \mathrm{CuO}_{2}$, exhibits a high- $T_{\mathrm{c}}$ of $110 \mathrm{~K}$ [14], we speculate that superconducting transitions are present in infinite-layer compounds composed of other transition metal cations, $A \mathrm{Ni}_{2} \mathrm{P}_{2}$ and $A \mathrm{Fe}_{2} \mathrm{P}_{2}$. Although to date, $A \mathrm{Fe}_{2} \mathrm{P}_{2}(A=\mathrm{Ca}, \mathrm{Sr}, \mathrm{Ba}, \mathrm{La})$ and $A \mathrm{Ni}_{2} \mathrm{P}_{2}(A=\mathrm{Ca}, \mathrm{La})$ have been examined, a superconducting transition has not been observed down to $1.8 \mathrm{~K}[12,15]$. Only $\mathrm{LaRu}_{2} \mathrm{P}_{2}$ with the $\mathrm{ThCr}_{2} \mathrm{P}_{2}$-type structure shows a superconducting transition at $4.1 \mathrm{~K}$ [15].

Here we report that a $\mathrm{ThCr}_{2} \mathrm{Si}_{2}$-type phosphide, $\mathrm{BaNi}_{2} \mathrm{P}_{2}$, exhibits a superconducting 
transition at $\sim 3 \mathrm{~K}$. Although Keimes et al. [16] have previously synthesized $\mathrm{BaNi}_{2} \mathrm{P}_{2}$ and reported its crystal structure, they did not report its electrical and magnetic properties. We synthesized $\sim 90 \%$ pure $\mathrm{BaNi}_{2} \mathrm{P}_{2}$ samples, and measured their electrical and magnetic properties down to 1.9 $\mathrm{K}$.

\section{Experimental}

Samples were prepared by a solid-state reaction of the starting materials, Ba (Johnson Matthey Company, 99.9\%), P (Rare Metallic, 99.9999\%), and Ni (Nilaco Corporation, 99.9\%). A stoichiometric mixture of the $\mathrm{Ba}, \mathrm{P}$, and Ni powders was pressed into a pellet, and heated in an evacuated silica tube initially at $400{ }^{\circ} \mathrm{C}$ for $12 \mathrm{~h}$ and then at $1000{ }^{\circ} \mathrm{C}$ for $12 \mathrm{~h}$. The sintered pellet was reground and subsequently pressed into a pellet, which was sintered at $1000{ }^{\circ} \mathrm{C}$ for $12 \mathrm{~h}$. The resulting samples were characterized by high-power X-ray diffraction (XRD, D8 ADVANCE-TXS, Bruker AXS) with $\mathrm{Cu} \mathrm{K} \alpha$ radiation, which detected trace amounts of impurity phases, $\mathrm{BaNi}_{9} \mathrm{P}_{5}, \mathrm{Ba}\left(\mathrm{PO}_{3}\right)_{2}$, and $\mathrm{BaNi}_{2}\left(\mathrm{PO}_{4}\right)_{2}$. Therefore, the crystal structure of $\mathrm{BaNi}_{2} \mathrm{P}_{2}$ and the compositions of the impurities were refined by the four-phase Rietveld method using the code TOPAS3 [17].

Electrical resistivity of the sintered pellets (apparent densities: $\sim 63 \%$ ) were measured in the temperature range from 1.9 to $300 \mathrm{~K}$ by the four-probe technique (using PPMS, Quantum Design). Sputtered Au films were used as ohmic contacts. Magnetic measurements were carried out using a vibrating sample magnetometer (VSM, using PPMS, Quantum Design). Temperature dependence of the magnetization was measured in a magnetic field at 10 Oe after zero-field cooling (ZFC) to the measurement temperatures.

\section{Results and discussion}

Figure 2 shows the powder XRD pattern of the purest sample obtained to date, which still shows diffraction peaks of $\mathrm{BaNi}_{2} \mathrm{P}_{2}, \mathrm{BaNi}_{9} \mathrm{P}_{5}, \mathrm{Ba}\left(\mathrm{PO}_{3}\right)_{2}$, and $\mathrm{BaNi}_{2}\left(\mathrm{PO}_{4}\right)_{2}$. Four-phase Rietveld analyses revealed that the obtained sample was mainly $\mathrm{BaNi}_{2} \mathrm{P}_{2}$, but contained $\sim 9$ vol.\% of $\mathrm{BaNi}_{9} \mathrm{P}_{5}, \sim 2$ vol.\% of $\mathrm{Ba}\left(\mathrm{PO}_{3}\right)_{2}$, and $\sim 1$ vol.\% of $\mathrm{BaNi}_{2}\left(\mathrm{PO}_{4}\right)_{2}$. The obtained samples are dark gray and chemically stable in air.

Figure 3 shows the temperature dependence of the electrical resistivity $(\rho)$ at an external magnetic field $(H)$ of 0 Oe. The resistivity at $300 \mathrm{~K}$ was $2.8 \mathrm{~m} \Omega \cdot \mathrm{cm}$, and a metallic behavior was observed at temperatures down to $\sim 3 \mathrm{~K}$. The inset shows a magnified view in the temperature range of $1.9-10 \mathrm{~K}$ as a function of $H$. At $H=0 \mathrm{Oe}, \rho$ dropped sharply at $\sim 3 \mathrm{~K}$, and the resistivity vanished at $2.7 \mathrm{~K}$, implying a superconducting transition. The onset temperature where $\rho$ begins to drop decreases as $H$ increases, and the drop in $\rho$ vanishes at $H=1000$ Oe. These results suggest that the observed changes are due to a superconducting transition at $\sim 3 \mathrm{~K}$.

Figure 4 shows the temperature dependence of the mass magnetization $(M)$ measured at 10 
Oe after the ZFC. $M$ was as small as $\sim 1 \times 10^{-4} \mathrm{emu} / \mathrm{g}$, and was nearly independent of temperature at $4-300 \mathrm{~K}$, implying Pauli paramagnetism in this temperature range. However, $M$ began to drop, became negative at $\sim 3 \mathrm{~K}$, and reached a large negative value of $-1.1 \times 10^{-1} \mathrm{emu} / \mathrm{g}$ at $1.9 \mathrm{~K}$. These results, together with the zero resistance in Fig. 3, clearly indicate that the obtained sample exhibits superconductivity at temperatures below $\sim 3 \mathrm{~K}$. The field dependence of the magnetization $(M-H)$ curve in the inset of Fig. 4 shows that the decrease in the negative magnetization is proportional to $H$ at $H<150 \mathrm{Oe}$, and then increases to zero as $H$ increases up to 550 Oe. This behavior is similar to that observed in type-II superconductors. Furthermore, in this case the lower and upper critical magnetic fields were estimated to be $H_{\mathrm{c} 1}=\sim 150$ Oe and $H_{\mathrm{c} 2}=$ $\sim 550$ Oe, respectively. The volume fraction of the superconducting phase estimated from the slope of the $M-H$ curve at $H<150$ Oe was nearly $100 \%$.

Next, the effects of the impurity phases were assessed. The impurity phase $\mathrm{BaNi}_{9} \mathrm{P}_{5}$ shows a temperature-independent Pauli paramagnetism [18], whereas $\mathrm{BaNi}_{2}\left(\mathrm{PO}_{4}\right)_{2}$ exhibits an antiferromagnetic transition at a Neel temperature of $24 \mathrm{~K}$ [19]. Rietveld analyses showed that the volume fractions of the impurity phases $\left[\sim 9\right.$ vol. $\%$ for $\mathrm{BaNi}_{9} \mathrm{P}_{5}, \sim 1$ vol. $\%$ for $\mathrm{BaNi}_{2}\left(\mathrm{PO}_{4}\right)_{2}$, and $\sim 2$ vol.\% for $\left.\mathrm{Ba}\left(\mathrm{PO}_{3}\right)_{2}\right]$ were negligible compared to that of the superconducting phase. Consequently, we conclude that $\mathrm{BaNi}_{2} \mathrm{P}_{2}$ is a bulk superconductor below $\sim 3 \mathrm{~K}$. Although other Ni-based superconductors, $L n \mathrm{Ni}_{2} \mathrm{~B}_{2} \mathrm{C}(L n=\mathrm{Y}, \mathrm{Tm}, \mathrm{Er}, \mathrm{Ho}$, and $\mathrm{Lu})$ and $\mathrm{La}_{3} \mathrm{Ni}_{2} \mathrm{~B}_{2} \mathrm{~N}$, which are composed of tetrahedral $\mathrm{Ni}$ layers similar to $\mathrm{LaNiOPn}$ and $\mathrm{BaNi}_{2} \mathrm{P}_{2}$, have been reported, each $\mathrm{Ni}$ ion is coordinated not by $P n$, but by B ions [20, 21]. It is known in crystal chemistry and complex chemistry that $\mathrm{Cu}^{2+}$ ions tend to take planar four coordinate structures, while $\mathrm{Ni}$ and $\mathrm{Fe}$ ions prefer to take tetrahedral structures, suggesting that a planar $\mathrm{Cu}^{2+}$ structure is not a requisite for superconductivity, and such a transition metal-based tetrahedral layer is key to discovering new superconductors.

\section{Summary}

$\mathrm{BaNi}_{2} \mathrm{P}_{2}$, which belongs to the $A T_{M 2} \mathrm{P}_{2}$ system with the $\mathrm{ThCr}_{2} \mathrm{Si}_{2}$ structure, is regarded as an infinite-layer structure. $\mathrm{BaNi}_{2} \mathrm{P}_{2}$ shows a superconducting transition at $\sim 3 \mathrm{~K}$. Thus, $\mathrm{BaNi}_{2} \mathrm{P}_{2}$ is tentatively assigned as a type-II superconductor with a lower critical magnetic field of $\sim 150$ Oe and an upper field of $\sim 550 \mathrm{Oe}$ at $1.9 \mathrm{~K}$. In the $A T_{M 2} \mathrm{P}_{2}$ system, the transition metal $T_{M}$ can be replaced with other transition metal ions, and the lattice parameters can also be controlled by replacing the $A$ cation. These features provide a new platform to systematically survey the relationship among superconducting transitions, $d$ electron configurations, and crystal structures. Hence, the discovery of higher- $T_{\mathrm{c}}$ superconductors is anticipated in this and related material systems. 


\section{References}

[1] A. Schilling, M. Cantoni, J. D. Guo,H. R. Ott, Nature 363 (1993) 56.

[2] Y. Maeno, H. Hashimoto, K. Yoshida, S. Nishizaki, T. Fujita, J. G. Bednorz, F. Lichtenberg, Nature 372 (1994) 532.

[3] K. Takada, H. Sakurai, E. Takayama-Muromachi, F. Izumi, R. A. Dilanian, T. Sasaki, Nature $422(2003) 53$.

[4] S. Yamanaka, K. Hotehama, H. Kawaji, Nature 392 (1998) 580.

[5] M. J. Geselbracht, T. J. Richardson, A. M. Stacy, Nature 345 (1990) 324.

[6] Y. Kamihara, H. Hiramatsu, M. Hirano, R. Kawamura, H. Yanagi, T. Kamiya, H. Hosono, J. Am. Chem. Soc. 128 (2006) 10012.

[7] Y. Kamihara, T. Watanabe, M. Hirano, H. Hosono, J. Am. Chem. Soc. 130 (2008) 3296.

[8] T. Watanabe, H. Yanagi, T. Kamiya, Y. Kamihara, H. Hiramatsu, M. Hirano, H. Hosono, Inorg. Chem. 46 (2007) 7719.

[9] T. Watanabe, H. Yanagi, Y. Kamihara, T. Kamiya, M. Hirano, H. Hosono, J. Solids State Chem. (2008), doi:10.1016/j.jssc.2008.04.033.

[10] H. Yanagi, R. Kawamura, T. Kamiya, Y. Kamihara, M. Hirano, T. Nakamura, H. Osawa, H. Hosono, (submitted).

[11] E. Mörsen, B. D. Mosel, W. Müller-Warmuth, M. Reehuis, W. Jeitschko, J. Phys. Chem. Solids 49 (1988) 785.

[12] W. Jeitschko, M. Reehuis, J. Phys. Chem. Solids 48 (1987) 667.

[13] M. Reehuis, W. Jeitschko, G. Kotzyba, B. Zimmer, X. Hu, J. Alloys Compd. 266 (1998) 54.

[14] M. Azuma, Z. Hiroi, M. Takano, Y. Bando, Y. Takeda, Nature 356 (1992) 775.

[15] W. Jeitschko, R. Glaum, L. Boonk, J. Solid State Chem. 69 (1987) 93.

[16] V. Keimes, D. Johrendt, A. Mewis, C. Huhnt, W. Schlabitz, Anorg. Allg. Chem. 623 (1997) 1699.

[17] Bruker AXS. TOPAS, version 3, Bruker AXS, Karlsruhe, Germany, 2005.

[18] J. V. Badding, A. M. Stacy, J. Solid State Chem. 87 (1990) 10.

[19] L. P. Regnault, J. Y. Henry, J. Rossat-Mignod, A. De Combarieu, J. Magn. Magn. Mat. 15-18 (1980) 1021.

[20] R. J. Cava, H. Takagi, H. W. Zandbergen, J. J. Krajewski, W. F. Peck Jr, T. Siegrist, B. Batlogg, R. B. Van Dover, R. J. Felder, K. Mizuhashi, J. O. Lee, H. Eisaki, S. Uchida, Nature 367 (1994) 252.

[21] R. J. Cava, H. W. Zandbergen, B. Batlogg, H. Eisaki, H. Takagi, J. J. Krajewski, W. F. Peck Jr, E. M. Gyorgy, S. Uchida, Nature 372 (1994) 245. 
(a)

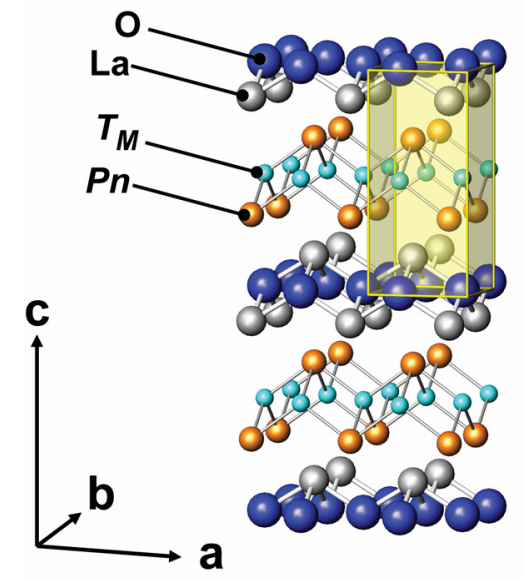

(b)

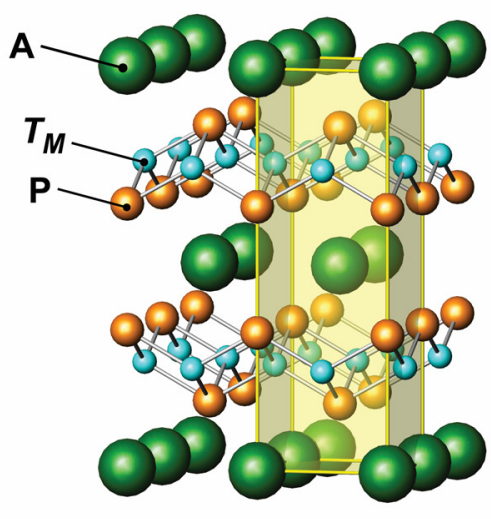

Fig. 1. (a) Crystal structure of $\mathrm{La} T_{M} \mathrm{O} P n$. La-O layers and $T_{M}-P n$ layers are stacked along the $c$ axis. (b) Crystal structure of $A T_{M 2} \mathrm{P}_{2}$. Structure of the $T_{M}$-P layer is similar to that in $\mathrm{La}_{M} \mathrm{OP}$. 


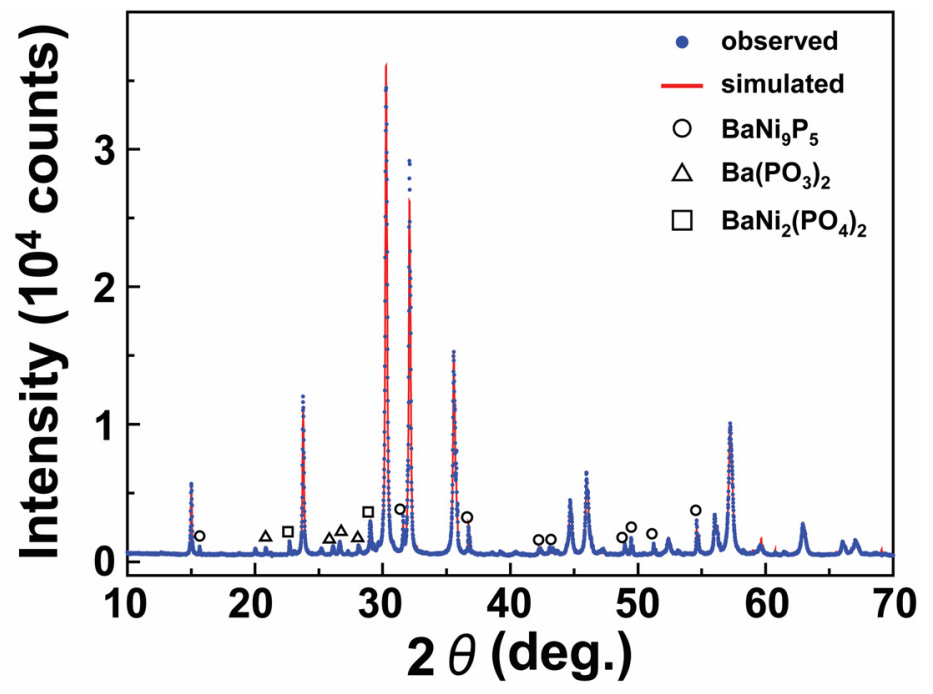

Fig. 2. XRD pattern of $\mathrm{BaNi}_{2} \mathrm{P}_{2}$ sample measured (blue circles) and simulated by the Rietveld method using the refined result (red line). Trace amounts of impurities, $\mathrm{BaNi}_{9} \mathrm{P}_{5}, \mathrm{Ba}\left(\mathrm{PO}_{3}\right)_{2}$, and $\mathrm{BaNi}_{2}\left(\mathrm{PO}_{4}\right)_{2}$, are detected, and their diffraction peaks are indicated by open circles, triangles, and squares, respectively. 


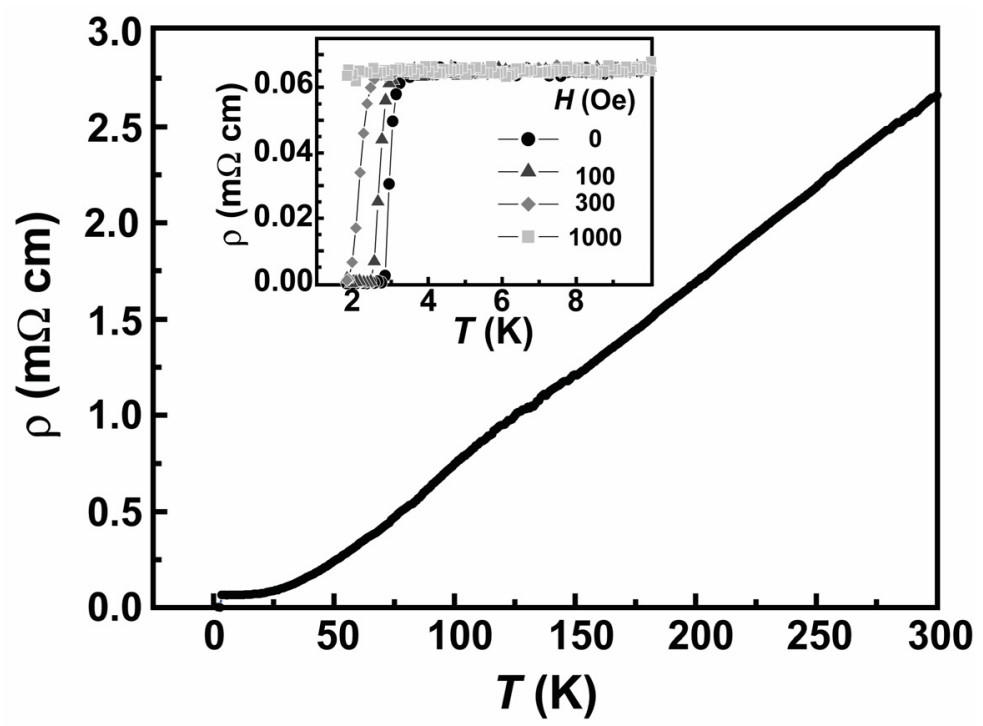

Fig. 3. Temperature $(T)$ dependence of the electrical resistivity $(\rho)$ at $H=0$ Oe. Inset shows the $\rho-T$ curves as a function of $H$ magnified in the temperature range of $1.9-10 \mathrm{~K}$. 


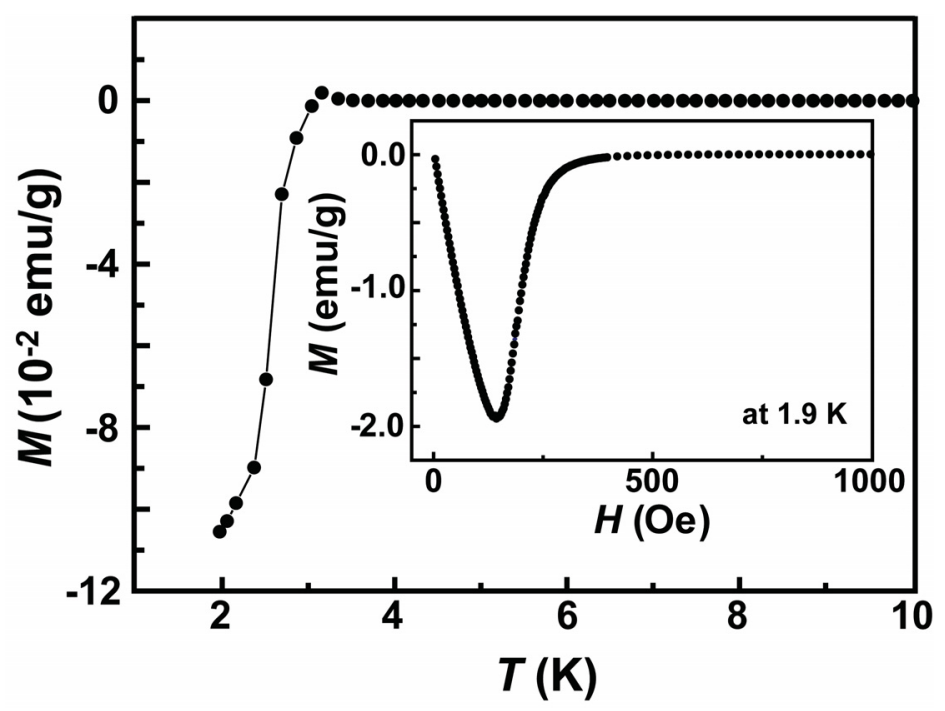

Fig. 4. Temperature $(T)$ dependence of the mass magnetization $(M)$ measured at 10 Oe after cooling to $1.9 \mathrm{~K}$ under a zero magnetic field. Inset shows the filed $(H)$ dependence of $M$ at $1.9 \mathrm{~K}$. 